\title{
The Geometry and Motion of Nematode Sperm Cells
}

\author{
Evgeny Demekhin \\ Moscow State University \\ Nicole Haugen \\ Bemidji State University \\ Brian Ibanez \\ Andrews University \\ Jerome Lederman \\ Preuss School, University of California, San Diego \\ Kaitlyn Murphy \\ Montclair State University \\ Diana Verzi \\ San Diego State University \\ Debra Witczak \\ Illinois State University
}

August 15, 2008

\begin{abstract}
The movement of Ascaris suum sperm cells is caused by protrusive, adhesive and contractile forces that act inside the cell. A discrete, 2-D geometric model of the forward motion and turning of the cell is modeled in this paper. The general assumption used is that the internal $\mathrm{pH}$ level of the lamellipod is responsible for motility. The main focus of this model is the protrusion of the front boundary of the cell, although contractile and adhesive forces are present in the model. This model only considers MSP bundles because they appear to be responsible for protrusion, and they make up the framework for the shape of the lamellipod.
\end{abstract}




\section{INTRODUCTION}

\subsection{Biological Background}

The motility of specific animal cells plays an integral role in many biological processes. For example, wherever an animal tissue may be damaged fibroblasts will crawl to the site and repair the damage. Tracking down foreign pathogens and eliminating them, along with the threat they present to the body, is the work of the motile neutrophils. However, not all motile cells are beneficial, such as in the case of metastatic cells. These cancer cells invade distant areas of the body enabling the cancer to spread. A thorough understanding of how animal cells are able to accomplish movement can be very applicable in the field of medicine. Therefore, there is considerable interest in modeling cellular motility.

All of these cells move by the same basic process of first protruding the front of the cell outward, adhering to the surface, and contracting at the rear. However, complications quickly arise when examining these processes more closely. In most eukaryotic cells the main players in cellular movement are actin and myosin [15]. In addition to providing the basis of cell motility, these structures also function in the process of phagocytosis, movement of vesicles and organelles, surface rearrangement, and cytokinesis. Actin and myosin require sets of accessory proteins for each specific activity creating an interconnected and complex system [12]. It becomes very difficult to untangle this complexity in order to determine what contributes to cell motility in this multi-functional cytoskeleton.

Rather than swimming with the assistance of flagella, the motile sperm of the nematode Ascaris suum crawl like amoebae with the aid of a motile appendage called a lamellipod [12]. These sperm cells, though achieving locomotion through the same cycle of protrusion, adhesion and contraction, have a simplifying characteristic that sets them apart from most crawling cells. Instead of using an actin/myosin network, the cytoskeleton of Ascaris suum consists of a Major Sperm Protein (MSP) gel network. The MSP cytoskeleton functions only in the process of cell locomotion; therefore, it avoids many of the complications associated with studying actin-based motility [12]. In addition, many cellular components such as the endoplasmic reticulum, the Golgi apparatus, and the usual actin cytoskeleton are left behind in the residual body after meiosis [3]. The result of this process is a cell that is dedicated almost entirely to locomotion, providing us with a relatively straightforward system for studying cell motility. Though simplified, the cytoskeletal dynamics of Ascaris suum still employ similar mechanical principles in order to generate motility as the more complex actin-based systems [13].

MSP Cytoskeleton In the cell lamellipod MSP is organized into 15-20 long branched fiber complexes (bundles) which extend backwards from the leading edge to the back of the cell. This network of crosslinking fiber complexes form the cytoskeleton. According to Sepsenwol and Taft, it is this cytoskeleton that shapes the lamellipod [14].

Protrusion: The process of protrusion in Ascaris suum is accomplished at the leading edge of the lamellipod by polymerization reactions which produce enough force to push the front of the cell forward [15]. Polymerization refers to the process in which additional MSP units are added on to subfilaments through a nucleation-elongation reaction [4]. At various locations along the leading edge of the lamellipod two proteins, a membrane protein (MSP Polymerization Organizing Protein or MPOP) and a cytosolic protein (MFP2), come together to create a 
nucleation complex (also referred to as a vesicle) which catalyzes the polymerization reaction $[5,16]$. Studies have shown that the rates of elongation of MSP subfilaments fall sharply at a distance of about $0.5 \mu \mathrm{m}$ from the nucleation complex, which indicates that polymerization only occurs in close vicinity to the vesicle $[5,6]$. As the newly polymerized subfilaments flow backwards from the leading edge and out of this elongation region they self assemble into the crosslinking fiber complex [5]. Interestingly enough, it is the concentration of the cytosolic protein rather then the amount of MSP that limits the rate of fiber growth [16]. In vitro experiments have shown that MSP polymerization will keep pace with the translocation of the nucleation complex [3]. This corresponds to MSP polymerization keeping pace with the rate of protrusion of the leading edge in Ascaris. Figure 1 identifies the key structures involved in the polymerization reaction.

Contraction: While protrusion is accomplished by MSP polymerization at the front of the cell, contraction is driven by the depolymerization of the assembled cytoskeletal network at the rear of the lamellipod. One protein has been identified, called Yersinia Enterocolytica Tyrosine Phosphatse (YOP), and this protein disassembles the bundles at the back of the cell into their individual polymerizing units [7]. In vitro experiments have led to the proposal of cytoskeletal depolymerization as a force-producing mechanism which pulls the cell body forward [17, 7, 15].

Adhesion: Adhesion, or the attraction of the lamellipod to the substrate, plays a very integral role in generating the forward motion of the cell. Adhesion is strong at the leading edge and diminishes toward the rear of the cell where practically no adhesion occurrs underneath the cell body. This is the critical property for cell motility since without it the processes of polymerization and depolymerization would only recycle the MSP cytoskeleton without accomplishing any forward motion [8]. Using this property, contractile forces pull up the adhesive sites at the rear of the cell while protrusion lays down additional adhesion sites at the front. This process results in a continuous cycle of attachment-detachment between the lamellipod membrane and the substrate which generates a forward motion [11].

$p H$ gradient: The interior $\mathrm{pH}$ seems to play a regulatory role in nearly every facet of motility in Ascaris. A pH gradient that decreases from the front of the cell to the rear is maintained by the cell with the leading edge being 0.2 units higher [9]. Though the molecular mechanism for adhesion has not yet been identified [17], it appears to be regulated in part by the $\mathrm{pH}$ since adhesion is stronger at a higher $\mathrm{pH}$ and weakens in the more acidic environment. The $\mathrm{pH}$ gradient also plays a role in the spatial separation of protrusion and contraction by acting as an on/off switch for the critical proteins involved in these processes. A tyrosine kinase phosphorylates MPOP (the membrane protein involved in protrusion) at the leading edge where the $\mathrm{pH}$ is higher, thereby activating the protein and enabling protrusion to occur [5]. Though not actively triggering cytoskeletal disassembly and retraction, the $\mathrm{pH}$ gradient may play an indirect role by activating a phosphatase that would in turn activate the protein involved in depolymerization, YOP [7]. Figure 2 shows the relationship between $\mathrm{pH}$ and the processes of protrusion, adhesion, and contraction. 


\subsection{Previous Mathematical Models}

In [10], Mogilner and Verzi proposed a 1-D model for the motility of the nematode sperm cell A. suum. They assumed the bundles, polymers and nodes affect the velocity of the cell's motion. The speed of motion was connected to these densities by the conservation of momentum (a moving mass). The stress was a function of all these densities along with the assumption of a viscous intracellular environment, giving them a relationship between the gradient of the stress, adhesion and the speed of motion. In this model the speed of motion at the front and at the back is dependent on the known functions imposed by the authors and created by observations. This approach allowed them to calculate the speed of motion of each point inside the cell body at each time step.

Mogilner and Verzi [10] assumed an arctan function for the rate of polymerization, depolymerization, and nodal adhesion over the length of the lamellipod. These assumptions lead to a velocity of protrusion given by:

$$
v_{p}(f(t))=A\left[0.5+\frac{1}{\pi} \arctan (\eta(f(t)-L))\right] \frac{L}{f(t)-r(t)}
$$

where $v_{p}$ is the rate of protrusion at the front of the lamellipod. The other variables apply to the geometry of the lamellipod; $f(t)$ is the coordinate of the front, $r(t)$ the coordinate of the rear and $\mathrm{L}$ is the distance from the rear of the cell to the critical $\mathrm{pH}$ value.

In [2], Choi, Lee and Lui proved existence and uniqueness of solutions to the equations using finite element methods in [10]. Under simplifying assumptions, the authors of [1] looked at a 2-D model and interpreted the cell as an elastic plate, but this approach has little in common with the modeling approach that we took.

They link the velocity of protrusion to $\mathrm{pH}$ and a maximum surface area of the lamellipod, assuming the maximum area is related to some depletable controlling factor. The formula used in this paper is:

$$
v_{p}(f(t))=v_{0} \bullet f(p H) \bullet \frac{A_{\max }-A}{A_{\max }}
$$

where $f(p H)$ is a linear function of $\mathrm{pH}$ over the length of the lamellipod.

\subsection{Thesis and Assumptions}

Our model begins to expand on the model proposed by Mogilner and Verzi to a second dimension that is parallel to the substratum. This model only considers the front edge of the cell, which is the front boundary condition, and uses a discrete model. We also choose a discrete model because the electron microscope observations show a very limited amount of bundles protruding in the lamellipod.

The nematode sperm cell's motion and contour are driven by the growth of its cytoskeletal elements, which, in turn, are regulated by a spatial pH distribution, 


\section{and the availability of membrane proteins.}

We assume:

- Filament protrusion occurs perpendicular to the contour of the leading edge.

- Throughout the lamellipod, the $\mathrm{pH}$ gradient is linearly related to its distance from the cell body with $\mathrm{pH} \approx 6.2$ at the cell body interface and $\mathrm{pH} \approx 6.4$ at the maximal extended length of the lamellipod.

- The pH gradient throughout the cell creates different rates of filament protrusion. The tips of filaments grow in the direction of motion quickly, while the tips near the cell body interface grow very slowly.

- The cell body is an ellipse.

- Due to the limited amount of vesicle proteins, there is an upper bound on the rate of polymerization.

- The cell body moves forward in a direction perpendicular to its major axis.

- The cell body's velocity is the average speed of bundle protrusion.

- Turning of the cell occurs by a rotation of the $\mathrm{pH}$ vector in response to a change in the external environment.

\section{METHODS}

In this section, the methods we used to construct the Matlab program are described, including the mathematical equations behind the model, the geometry that governs the program, and a detailed description of the program itself.

\subsection{The Mathematics of Protrusion}

The cell is composed of a body lying on a lamellipod that is composed of a series of rigid filaments, cytoskeletal bundles, that are held together by a passive gel. The cell is dragged forward by cytoskeletal protrusion in combination with the forces transmitted by the adhesive interaction of hydrophobic nodal points and the cell sub-stratum. This happens simultaneously with contractive action resulting from dissolving cytoskeletal bundles at the lamellipod-cell body interface. Our model's rate of protrusion is described by a differential equation of the form:

$$
\frac{\partial \overrightarrow{r_{i}}}{\partial t}=v_{i} \overrightarrow{n_{i}}
$$


where $r_{i}$ is the position vector of cytoskeletal element $i, v_{i}$ is the velocity of protrusion and $\overrightarrow{n_{i}}$ is a vector in the perpendicular direction to the lamellipod contour for each cytoskeletal element $i$. For purposes of the Matlab simulation, we discretize the differential equation as follows:

$$
\vec{r}_{t+1_{i}}=\vec{r}_{t_{i}}+v_{t_{i}} \vec{n}_{t_{i}} \tau
$$

where $\tau$ is the timestep. Notice that we introduce the notation $z_{t_{i}}$ where $z$ represents the variable under consideration, $t$ represents the time step and $i$ represents the bundle number (note that the typical number of bundles present at any given time is between 15 and 20 [4]). We assume the cytoskeleton bundles dissolve and the fibers de-polymerize as a result of the more acidic conditions near and under the cell body. Thus we make the connection between contraction and $\mathrm{pH}$. The protrusion of the leading edge of the lamellipod is also controlled by $\mathrm{pH}$. The higher $\mathrm{pH}$ is necessary for the activation of the vesical protein that is lodged in the lamellipod membrane. Since $\mathrm{pH}$ varies approximately linearly [1] with distance from the cell body interface, we describe $\mathrm{pH}$ as a function of distance:

$$
p H_{t_{i}}\left(x_{t_{i}}\right)=6.2+\frac{x_{t_{i}}}{l(t)}
$$

where $x_{t_{i}}$ is the distance from the cell body of a particular cytoskeletal element and $l(t)$ is the fixed length of the lamellipod at time $t$ ( $l$ is a limiting factor for $\mathrm{pH}$ ). This functions gives us values of internal $\mathrm{pH}$ from 6.2 (at the back of the cell where $x_{t_{i}}=0$ ) to approximately 6.4 (near the front of the cell).

While the $\mathrm{pH}$ varies linearly over the length of the lamellipod, the protrusion speed is governed by a chemical process whose rate optimizes at a particular $\mathrm{pH}$. In the program, we use the function of the form:

$$
\begin{gathered}
v_{t_{i}}\left(x_{t_{i}}\right)=v_{0} \quad \text { for } 0 \leq x_{t_{i}} \leq L_{0} \\
v_{t_{i}}\left(x_{t_{i}}\right)=\gamma x_{t_{i}} e^{\left(-\lambda x_{t_{i}}^{2}\right)} \quad \text { for } x_{t_{i}}>L_{0}
\end{gathered}
$$

where $L_{0}$ is some maximum constant. The rate of protrusion is a constant rate $v_{0}$ until the lamellipod is fully extended and capable of pulling the cell body forwarf. Note that $\gamma$ is a constant that affects the maximum velocity and $\lambda$ is a decay rate. Solving (5) for $x_{t_{i}}$ and plugging this into $(7)$, we have: $\mathrm{v}_{t_{i}}\left(p H_{t_{i}}\right)=5 \gamma l(t)\left(p H_{t_{i}}-6.2\right) e^{-25 \lambda l(t)\left(p H_{t_{i}}-6.2\right)^{2}}$ and so velocity of protrusion of each cytoskeletal filament $i$ is a function of $\mathrm{pH}$.

The direction of motion is governed by the contraction of the lamellipod at the cell bodylamellipod interface. After the lamellipod reaches a predefined length, equal to the width of the cell body, we assume the cell body moves in the direction of its $x$-axis, at the average speed of the skeletal filaments.

Rotation of the cell is assumed to occur due to a rotation of the $\mathrm{pH}$ gradient brought on by some external event. This altered $\mathrm{pH}$ gradient results in an immediate realignment of the cell body with the $\mathrm{pH}$ gradient, i.e. the cell body rotates and the direction of its velocity is in the direction of the $\mathrm{pH}$ gradient. Rotation of the axes of the cell body alters the calculation of $x_{t_{i}}$, which in turn, alters $v_{t_{i}}$, which in turn alters the shape of the lamellipod. Thus the cell turns in the direction of the $\mathrm{pH}$ gradient. 


\subsection{The Geometry of Protrusion}

We assume the cell body resembles an ellipse with its major axis being parallel to the y axis. Therefore, the initial condition of our model is simply the equation of an ellipse:

$$
\frac{x^{2}}{k^{2}}+y^{2}=1
$$

where $k$ is a constant that determines the shape of the ellipse. We determine the initial points on the ellipse by dividing $\pi$ (the angle realized when moving clockwise from $\pi / 2$ to $-\pi / 2$ ) into equal angles determined by the number of bundles. Using these angles we connect rays from the origin to the initial ellipse and these rays have the form $y_{0_{i}}=\tan \left(\theta_{0_{i}}\right) x_{0_{i}}$, where $\theta_{0_{i}}$ is the angle on the ellipse where bundle $i$ will start growing. Plugging $y_{0_{i}}$ into (8) and solving for $x_{0_{i}}$ we have $x_{0_{i}}=\frac{k}{\sqrt{1+k^{2} \tan ^{2}\left(\theta_{0_{i}}\right)}}$. This gives us the coordinates where bundles will begin to grow from the initial ellipse. We then desire to find the coordinates for subsequent points according to our difference equation. In order to accomplish this, we approximate the slope of the line perpendicular to the contour at each point (this is motivated by our normal growth assumption) as follows:

$$
m_{t_{i}}=\frac{x_{t_{i+1}}-x_{t_{i-1}}}{y_{t_{i-1}}-y_{t_{i+1}}}
$$

for each time $t>0$ and bundle $i$. We then caculate the cosine and sine this slope makes with the $\mathrm{x}$-axis as follows:

$$
\begin{aligned}
& \cos \left(\theta_{t_{i}}\right)=\frac{1}{\sqrt{1+m_{t_{i}}^{2}}} \\
& \sin \left(\theta_{t_{i}}\right)=\frac{m_{t_{i}}}{\sqrt{1+m_{t_{i}}^{2}}}
\end{aligned}
$$

where $\theta_{t_{i}}$ is the angle that the slope makes with the x-axis at time $t>0$ for bundle $i$. We are then ready to use the difference equation (4) which can be expressed in terms of $x$ and $y$ coordinates as follows:

$$
\begin{aligned}
& x_{(t+1)_{i}}=x_{t_{i}}+v_{t_{i}} \cos \left(\theta_{t_{i}}\right) \tau \\
& y_{(t+1)_{i}}=y_{t_{i}}+v_{t_{i}} \sin \left(\theta_{t_{i}}\right) \tau
\end{aligned}
$$

where $\tau$ is the timestep. This process is repeated the duration of the simulation unless a bundle is depolymerized. Because we have a fixed number of bundles at any given time (from the biolgical observation that there is a fixed number of bundles throughout time [14]), if a bundle is depolymerized, one must be added at some point on the contour of the lamellipod. Our simulation adds a bundle growth point where the distance between adjacent bundles is the greatest (because membrane proteins are uniformly distributed throughout the lamellipodial membrane). To accomplish this we use the standard parabolic interpolation technique using three known points. The three known points include the two points that have the greatest adjacent distance, and one other point adjacent to the two points. We chose parabolic interpolation in order to maintain the curvature of the lamellipod. However, other spline techniques may have also been used to maintain curvature.

These equations govern the geometry through time of the nematode sperm with the assumption of the normal growth and an assumed velocity function. 


\subsection{Matlab Simulated Program}

We now provide a detailed description of the Matlab program, which includes a computer flow chart (Figure 4), to visually represent the processes of the Matlab simulation. This stepby-step description allows the user to understand the inputs and outputs of the program, and the resulting movies that follow.

1. In the first step the program receives the following information from the user:

- The amount of time units to run the program. (One time unit represents about one minute and the motion of about one cell body length)

- The initial shape of the cell body.

- The angle and time at which a turn should occur.

- The number of bundles in the lamellipod.

2. The program initializes the shape of the cell body by plotting the coordinates for filament polymerization.

3. The program checks if it is time for the cell body to start moving. (The program waits for the lamellipod to extend to approximately the size of the cell body )

4. When it is time for the cell body to begin moving, the program calculates the $\mathrm{pH}$.

5. The $\mathrm{pH}$ at each filament's tip and the placement of the cell body is used to calculate the velocity at each filament's tip.

6. The lamellipod is translated in the direction of the $\mathrm{pH}$ gradient.

7. The program checks to add filaments. (It uses the conservation of bundles rule, so if one bundle stops growing, then another one starts at the place where the distance between the filaments' tips is the greatest)

8. (a) If a filament needs to be added, the program interpolates (using quadratic interpolation) the cell body's front curve and adds a filament between two others on the curve. Then it checks if more filaments need to be added.

(b) If not, then it checks if the program has expired.

i. If yes, the program stops.

ii. If no, it loop back to calculating the $\mathrm{pH}$ values at each of the filament tips.

\section{RESULTS}

We simulated the evolving geometry of a moving nematode sperm cell, which includes extension of the lamellipod, cell body motion, turning at some pre-determined angle of rotation, and steady state cell body shape. Our program is guided by a set of assumptions and observations that were developed by observing videos of crawling nematode sperm cells developed 
by previous authors that have studied these cells. A graphical model that demonstrates these events through time consistent with the videos from laboratory experiments is the main result of this program. Our assumptions and observations that guide the framework of this program are listed in Section 1.3.

The dynamics of the program can be divided into four separate processes. The program first extends the lamellipod away from the cell body, with no movement of the cell body. After the lamellipod has extended to approximately the length of the cell body, cell movement begins. If specified, the cell body turns at a pre-determined timestep to a pre-determined angle. Finally, the program achieves a unique steady state cell shape for every programmed initial condition.

\subsection{Extending the Lamellipod}

The user specifies the number of bundles to be maintained over time. At each moment in time, several points, which correspond to the number of bundles, are added to the graph of the cell. These points represent the positions of the bundles resulting from protrusion. Each bundle has a unique protrusion rate which is calculated by the velocity function based on the distance from the cell body. Each bundle also has a unique direction of growth which is normal to the contour of the lamellipod. These features of the program result in noticeable curvature of the bundles as they grow through time. This visual result is consistent with laboratory video and images present in the previous literature.

\subsection{Moving Body}

The second process of the program includes a movement of the cell body. Biologically, it was determined that movement of the cell body occurs because of the forces transmitted by the adhesive interaction of the hydrophobic nodal points and the cell substratum and contractive action resulting from dissolving cytoskeletal bundles at the lamellipod-cell body interface. Our program assumes movement once the length of the extension of the lamellipod is approximately equal to the length of the cell body.

According to Roberts and King [11] only the fiber complexes in a limited sector at the leading edge of the lamellipod undergoes continuous elongation. Fibers outside that region become displaced toward the side and eventually stopped elongating as the cell moves forward over them. Those fibers that extended towards the center of the leading edge persist as long as the cell moves forward in a straight line. Both of these cytoskeletal characteristics were apparent in our computer simulation

\subsection{Turning}

According to in vitro observations of sperm cells, in order to execute a turn, a bulge is formed on the side of the lamellipod which then took over as the leading edge. This was accomplished by the cell encouraging cytoskeletal growth where the new leading edge was to be and arresting growth at the previous leading edge. Other experiments have had similar observations as well [12], [14]. Our model program seems to be consistant with these observations. At a predetermined time and angle, contraction rotates the body and a bulge is initiated at the opposite 
side. ( see figure 5D) This simulation was consistent with experimental observations that the formation of fiber complexes are integral in determining the location of surface protrusion and thereby the direction of motion [12].

\subsection{Steady State Cell Body Shape}

In figures 6 and 7 the model extends the lamellipod and initiates cell body motion (horizontal axis) and a constant steady state shape is formed. Figures 8 and 9 illustrate a consistant lamellipod shape over time and space. In figure 11 the same steady state shape is reached regardless of the initial cell body shape.

\section{DISCUSSION}

We, in our analysis, expand upon [1] and [10] to create a 2-dimensional approach to the crawling cell problem that can be solved by a finite difference scheme rather than the finite element approach. The nematode sperm cell can be described as a cell body sitting atop a very flat lamellipod. The cell body accounts for approximately half the lamellipod. Inside the lamellipod MSP polymerization occurs, polymerized MSP interweaves to form fibers, dimers or filaments of rest length $\lambda$, and these fibers interweave to form bundles of rest length $\rho$. The bundled elements have regularly spaced hydrophobic locations, nodes, which viscously attach to the cell substratum. The bundles "arch", curve along their length, from the cell body to the outer contour of the lamellipod. Multiple bundles, linked serially, and their nodes constiute a single cytoskeletal element. A typical nematode sperm cell consists of 15 - 20 cytoskeletal elements.

We choose to describe the sperm cell in cartesian coordinates, which we relate to curvilinear coordinates when describing the behavior of individual cytoskeletal elements. The variable $\mathrm{x}$ describes the typical direction of motion of the sperm cell and the variable $y$ is perpendicular to $\mathrm{x}$ in the plane of the lamellipod. As the lamellipod is extremely thin compared to its other dimensions we neglect the direction perpendicular to the $\mathrm{x}-\mathrm{y}$ plane. In the $\mathrm{x}-\mathrm{y}$ plane the variable $\mathrm{s}$ describes points along a cytoskeletal element, the variable $\mathrm{u}$ describes coordinates perpendicular to a cytoskeletal element, and the variable $\theta$ describes the angle the tangent to the cytoskeletal element makes with the $\mathrm{x}$-axis.

Four variables are chosen to describe the lamellipods biology:

- The quantity of bundles along the length of the cytoskeletal element, $b(s, t)$. We express this variable in an unusual fashion, as the length of bundles per unit length of cytoskeleton. Thus, for example, we might have $500-\mu \mathrm{m}$ of filaments per $\mu \mathrm{m}$ of cytoskeleton. Using these units allows us to mathematically interpret a conversion of bundles to fibers without concern for a conversion factor.

- The quantity of free filaments along the length of the cytoskeletal element, $p(s, t)$. We also express this variable as $\mu \mathrm{m}$ of free filaments per $\mu \mathrm{m}$ of cytoskeleton. 
- The frequency (or density) of cytoskeletal nodes, $c(s, t)$. We express this variable as nodes per $\mu \mathrm{m}$ of cytoskeleton.

- The quantity of dimer, $d(t)$. We introduce this variable to maintain a closed loop on the mass of material.

Whereas $b(s, t), p(s, t)$ and $d(s, t)$ have units of length per unit length, they really represent an amount of material along a unit length of lamellipod and thus they are subject to a conservation of mass criteria. The same reasoning applies to $c(s, t)$.

Conservation Relations: We can express a conservation condition for each cytoskeletal element. Namely,

$$
\begin{gathered}
\frac{\partial b}{\partial t}=-\nabla \bullet(\vec{v} b)-\gamma_{b}(s) b \\
\frac{\partial p}{\partial t}=-\nabla \bullet(\vec{v} p)+\gamma_{b}(s) b-\gamma_{p}(s) p \\
\frac{\partial d}{\partial t}=-\nabla \bullet(\vec{v} d)+\gamma_{p}(s) p \\
\frac{\partial c}{\partial t}=-\nabla \bullet(\vec{v} c)
\end{gathered}
$$

The first term on the right side of each of equations (14) - (17) express the transport of material due to velocity $\vec{v}$. The remaining terms express the conversion of bundles into filaments, which occurs along the length of the lamellipod; filaments into bundles, which occur over a small region near the front edge of the lamellipod; and filaments into dimers, which principally occurs near and under the cell body. This closed loop process is completed by the diffusion of the dimers throughout the lamellipod. Note if we sum equations (14) - (17) we obtain,

$$
\frac{\partial(b+p+d)}{\partial t}=-\nabla \bullet(\vec{v}(b+p+d))
$$

Equation (18) corroborates the conservation of mass by specifying that the only change in the sum of the products of MSP and MSP over time is due to the mechanical transport of material.

The functions that describe the assembly rates, $\gamma_{b}(s)$ and $\gamma_{p}(s)$, are somewhat arbitrarily chosen to express known biological processes. We assume,

$$
\begin{gathered}
\gamma_{p}=\alpha_{p} \cdot\left(1-e^{-\left(\frac{x-L_{c}}{L_{c}}\right)^{2}}\right) \\
\gamma_{b}=\alpha_{b} \cdot e^{-\left(\frac{L_{c b}-x}{L_{c b}}\right)^{2}}
\end{gathered}
$$

where $L_{c b}$ is the length of the cell body and $L_{c}$ is the length of the cell.

Equation (19) expresses that the rate of solation of fibers into dimers is zero at the front edge of the lamellipod, where $x=L_{c}$, and rises monotonically moving in the direction of the cell body. Equation (20) expresses that the rate of unbundling is maximum at the front of the cell body. 
Stress-Displacement Relations: We next consider force displacement relations. The force developed in a single cytoskeletal element is the sum of the elastic, entropic force due to the elongation of the bundles and the elastic, entropic force due to the elongation of the filaments. This is expressed by,

$$
\sigma_{s}=K b(s, t)\left(\frac{1}{c(s, t)}-\rho\right)+\kappa p(s, t)\left(\frac{1}{c(s, t)}-\lambda\right)
$$

We assume the cytoskeletal element can only support force along its length. Forces perpendicular to its tangent line are transmitted to the viscous nodes. Here $\mathrm{K}$ is the effective bundle spring constant, $\kappa$ is the free filament spring constant, $\rho$ is the zero force length of a bundle, and $\lambda$ is the zero force length of the free filament. Since $c(s, t)$ is the node density, $1 / c(s, t)$ is the distance between nodes. When this is multiplied by the bundle density we get the length of the stretched bundle.

Equilibrium considerations plus the properties of the nodes dictate an additional relation. The force absorbed by the node is the result of the gradient of stress across the node. If we make a continuity assumption (which we have been making all along) and we also assume viscous behavior of the node we get,

$$
\nabla \sigma=\xi(s) \vec{v},
$$

where $\xi(\mathrm{s})$ is the effective drag coefficient for the node at location s and $\sigma$ is the magnitude of the force, which has components $\sigma_{s}$ and $\sigma_{u}$. The relates to the bundling rate. Thus we assume the same functional form over space,

$$
\xi=\alpha_{\xi} \cdot\left(1-e^{-\left(\frac{L_{c b}-x}{L_{c b}}\right)^{2}}\right)
$$

Impact of $\mathrm{pH}$ on Forward Velocity: Past models, whether 1-D or 2-D assumed a specific velocity vector for the motion of the sperm cell. Essentially researchers assumed the sperm cell moved in a uniform horizontal direction. While this might be true for the cell body as a whole, we make no such assumption for any particular cytoskeletal element. We assume that every cytoskeletal element has a growth rate that is perpendicular to the sperm cell contour at every moment in time and that the magnitude of growth rate is dependent upon the $\mathrm{pH}$ at the point where the cytoskeletal element meets the boundary of the cell. The growth rate conditions can be written,

$$
\vec{v}_{p}(s, t)=V_{\vec{r}(s, t)} \vec{n}
$$

where $\vec{v}_{p}(s, t)$ is the projection velocity at the front of the cytoskeleton element, $\vec{r}(t)$ is a position vector that prescribes the lamellipod contour, and $\vec{n}$ is the normal to the lamellipod contour. The magnitude of this velocity is a function of the local $\mathrm{pH}$ and that function is given by the diffusion equation [10] for hydrogen ionization,

$$
\frac{\partial H^{+}}{\partial t}=D \nabla^{2} H^{+}-\lambda\left(H^{+}-H_{e x t}^{+}\right)
$$

Without loss of generality, we assume hydrogen ion diffusion is rapid and steady state conditions exit. 
Mogilner and Verzi [10] estimate that the $\mathrm{pH}$ gradient from the front of the cell body to the front of the cell is constant and the $\mathrm{pH}$ beneath the cell body is a constant low value due to proton discharge from mitochondria located within the cell body. Bottino et al [1] cite "a depletable factor which limits the size of the lamellipod. They particularly note the dilution of the vesicle protein in the leading edge of the lamellipod as a limitiing factor for its growth. They also note other likely candidates that might limit growth. On this basis they assume that lamellipod area is limited to a maximum area. Thus higher $\mathrm{pH}$ at the lamellipod boundary and a lower density of vesicle protein (or some other depletable chemical) are offsetting factors that cause and constrain growth. We assume MSP assembly occurs above a $\mathrm{pH}$ value, $p H_{h}$ and assembly stops when the lamellipod perimeter reaches a specific value, $p_{\max }$. We use the formula,

$$
V_{p}=V_{0} f(p H) \max \left(1-\frac{p}{p_{\max }}, 0\right)
$$

where for each cytoskeletal element $V_{p}$ is the velocity of protrusion at the front of the lamellipod and $f(p H)$ is a function of $\mathrm{pH}$ such that $\mathrm{pH}$ varies linearly by 0.20 from rear to front of the lamellipod while $f$ varies linearly from 0 to 1 .

The Sperm Cell Model: We now finalize our description of the nematode sperm cell model. Up till this point we only referred to a single cytoskeletal element, which is a series of serially linked bundles that emanate from the front of the cell body through to the front contour of the lamellipod. The lamellipod consists of 15-20 such cytoskeletal elements, each of whose behavior is governed by the above equations. The forward movement of the front of the lamellipod is driven by projection of each cytoskeletal element normal to the contour at the elements location plus an additional motion due to the forces built up within the cell. Thus the forward velocity is a vector function of location along the contour. Certain elements, those away from the most forward position, will have no forward motion due to cytoskeletal projection since their most forward point is on the contour where the $\mathrm{pH}$ is above the critical value for maximal protrusion rate. Others, those in the most forward positions and lying within the critical $\mathrm{pH}$ range, will be growing perpendicular to the contour. Those cytoskeletal elements whose most forward positions lie below the lower critical $\mathrm{pH}$ value protrude slower than the maximal rate. And those nearest the cell body where the $\mathrm{pH}$ is below the disassembly value with disassemble. All the cytoskeletal elements eventually approach the cell body and decompose into unbound MSP filaments. Contraction occurs at the cell body, which is carried forward by this disassembly. Unbound MSP filaments re-circulate to the front of the sperm cell and provide the raw materials for additional filament bundling. The interaction of bundling and $\mathrm{pH}$ sensitivity results in a different curvature for each cytoskeletal element. The curvature is greatest for elements that reach the contour boundary nearest the cell body and least for elements that reach the contour boundary furthest from the cell body. While each element may be described by one-dimensional behavior, the interaction with $\mathrm{pH}$ creates a pseudo-two-dimensional effect, which could simulate turning behavior under the right driving forces. Equations 20 and 19 represent cytoskeletal protrusion rates that are consistent with these assumptions.

In steady state motion of the cell, the rate of filament and bundle polymerization must equal to the rate of filament and bundle depolymerization. Since polymerization results in motion 
of the forward boundary and depolymerization in forward motion of the rear boundary, equal rates of these processes should result in equal velocities of protrusion and contraction.

Thus we assume for steady state motion,

$$
V_{c}=\operatorname{Average}\left(V_{p}\right)
$$

where $V_{c}$ is the velocity of contraction of the cell body and that steady state motion has occurred when equation 26 is true.

Mogilner and Verzi [10] state that $\mathrm{pH}$ may be a factor effecting disassembly but other factors such as membrane tension and turnover of MSP polymers might also play a role. Thus they leave open the cause and assume in steady state motion a disassembly rate that is a function of location and a contraction velocity at the cell rear equal to the protrusion velocity at the cell front.

Boundary Conditions: Let the boundary contour of the sperm cell be defined by the vector $\vec{X}(x, y ; t)$, which is the position vector to a point on the sperm cell from some arbitrary origin. Call $\vec{f}=\vec{X}$ at the front boundary of the cell and $\vec{r}=\vec{X}$ at the rear boundary of the cell. The velocity of the boundary contour is governed by (1) cytoskeletal element bundle assembly or disassembly and (2) by the motion caused by the stresses built up and released through the polymerization and bundling processes. Thus we have,

$$
\left\{\begin{array}{l}
\frac{d \vec{f}}{d t}=\vec{v}_{p}(x, y, t)+\vec{v}(\vec{r}(t)) \\
\frac{d \vec{r}}{d t}=\vec{v}_{d}(x, y, t)+\vec{v}(\vec{r}(t))
\end{array}\right.
$$

The lamellipod is bounded by the cell boundary and a free boundary. At the free boundary,

$$
\begin{aligned}
\sigma_{s}(\vec{f}(t)) & =\sigma_{s}(\vec{r}(t))=0 \\
\sigma_{u}(\vec{f}(t)) & =\sigma_{u}(\vec{r}(t))=0
\end{aligned}
$$

The conditions on $b(s, t), p(s, t), d(s, t)$ and $c(t)$ on the boundary are derived from the following assumptions:

- Dimers immediately convert to filaments at the front boundary.

- There is zero flux of bundles, filaments and dimers at the boundary.

- The rear boundary is node free.

These assumptions result in the equations:

$$
\begin{gathered}
d(f(t))=0 \\
\nabla b(f(t)) \bullet \vec{n}=\nabla p(f(t)) \bullet \vec{n}=\nabla d(f(t)) \bullet \vec{n}=0 \\
(r(t))=0
\end{gathered}
$$

Equations in Coordinate Notation: As previously stated we introduce the x-y rectangular coordinates, coordinates fixed in space, and the s-u rectangular coordinates, local coordinates 
tangent and perpendicular to every point in each cytoskeletal element. Thus s-u rotated coordinates that are linked to $\mathrm{x}-\mathrm{y}$ through the angle that the cytoskeleton element makes with the x-axis, namely, $\theta$, which is a function of the cytoskeletal element and the location on that element. For any vector, $\vec{a}$, the divergence of $\vec{a}$ in terms of its s-u coordinates is given by,

$$
\nabla \bullet \vec{a}=\frac{\partial a_{s}}{\partial s}+\left(a_{s}-a_{u}\right) \frac{\partial \theta}{\partial s}
$$

Applying this form to the Conservation Equations,

$$
\begin{gathered}
\frac{\partial b}{\partial t}=-\left(\frac{\partial b v_{s}}{\partial s}+b\left(v_{s}-v_{u}\right) \frac{\partial \theta}{\partial s}\right)-\gamma_{b}(s) b \\
\frac{\partial p}{\partial t}=-\left(\frac{\partial p v_{s}}{\partial s}+p\left(v_{s}-v_{u}\right) \frac{\partial \theta}{\partial s}\right)+\gamma_{b}(s) b-\gamma_{p}(s) p \\
\frac{\partial m}{\partial t}=-\left(\frac{\partial m v_{s}}{\partial s}+m\left(v_{s}-v_{u}\right) \frac{\partial \theta}{\partial s}\right)+\gamma_{p}(s) p \\
\frac{\partial c}{\partial t}=-\left(\frac{\partial c v_{s}}{\partial s}+c\left(v_{s}-v_{u}\right) \frac{\partial \theta}{\partial s}\right)
\end{gathered}
$$

The vector equation describing the viscous node is restated as,

$$
\begin{gathered}
\frac{\partial \sigma_{s}}{\partial s}=\xi(s) v_{s} \\
\frac{\partial \sigma_{u}}{\partial s}=\xi(s) v_{u}
\end{gathered}
$$

\subsection{Conclusion}

The mathematical simulations for the protrusion and the turning of the cell did give a reasonable shape, although it modeled only the first few time steps of the turn. It turned out that no matter what initial shape of the cell was, in a few minutes the shape stabilized to a steady state and the steady state shapes for different initial body shapes were the same. Instead of conserving the total amount of MSP we conserve the total length of bundled MSP and the amount of bundles and this is the main difference with the other models as well as the fact that we are looking at each one of the filaments separately instead of looking at the meshwork of filaments.

Movies of the Matlab simultation demonstrating translation and rotation can be found on the webpage of the sixth author at http://www-rohan.sdsu.edu/ dverzi/ 


\subsection{Next Steps}

In this paper, we model geometrically the motion of a nematode sperm cell using the assumptions stated in the previous section. We are not completely satisfied with the result of the computer program. The cell body is only able to turn for small angles of $\theta$. Although this result is biologically satisfying, mathematical justification is needed to understand why the cell can successfully turn for only small values of $\theta$.

In order to complete the model, modifications need to be made to the differential equations used to model the movement of nematode sperm cells in previous papers, and these equations need to be incorporated into our geometric model.

The open questions that still remain include:

- What is the stimulus that keeps the cell moving?

- What external environmental effect triggers movement?

- Why do the bundles protrude perpendicular to the local contour?

- What is the chemical/biological connection between $\mathrm{pH}$ and protrusion?

- Is the exponential function for cytoskeletal growth reasonable or must we handle the growth causing and growth inhibiting factors separately?

- What is the connection between protrusion and contraction, if any?

\section{Acknowledgements}

The authors would like to thank Charles Wolgemuth and Mark Zajac from the University of Connecticut Health Center for providing useful movies that demonstrate the crawling of nematode sperm cells. The first five authors and the last author would also like to thank the sixth author for her mentorship and support on this research. This research was supported by the National Science Foundation REUT grant 0647384 at San Diego State University.

\section{References}

[1] D. Bottino, A. Mogilner, T. Roberts, M. Stewart, and G. Oster. How nematode sperm crawl. Journal of Cell Science, 115(2):367-384, 2002.

[2] YS Choi, J. Lee, and R. Lui. Traveling wave solutions for a one-dimensional crawling nematode sperm cell model. J Math Biol, 49(3):310-28, 2004.

[3] J.E. Italiano Jr, T.M. Roberts, M. Stewart, and C.A. Fontana. Reconstitution in vitro of the motile apparatus from the amoeboid sperm of Ascaris shows that filament assembly and bundling move membranes. Cell, 84(1):105-114, 1996. 
[4] JE Italiano Jr, M. Stewart, and TM Roberts. How the assembly dynamics of the nematode major sperm protein generate amoeboid cell motility. Int Rev Cytol, 202:1-34, 2001.

[5] L.L. LeClaire, M. Stewart, and T.M. Roberts. A 48 kDa integral membrane phosphoprotein orchestrates the cytoskeletal dynamics that generate amoeboid cell motility in Ascaris sperm. Journal of Cell Science, 116(13):2655-2663, 2003.

[6] L. Miao, O. Vanderlinde, J. Liu, R.P. Grant, A. Wouterse, K. Shimabukuro, A. Philipse, M. Stewart, and T.M. Roberts. The role of filament-packing dynamics in powering amoeboid cell motility. Proceedings of the National Academy of Sciences, 105(14):5390, 2008.

[7] L. Miao, O. Vanderlinde, M. Stewart, and T.M. Roberts. Retraction in Amoeboid Cell Motility Powered by Cytoskeletal Dynamics, 2003.

[8] A. Mogilner. Mathematics of cell motility: have we got its number? J Math Biol, 2008.

[9] A. Mogilner and G. Oster. CELL BIOLOGY: Enhanced: Shrinking Gels Pull Cells, 2003.

[10] A. Mogilner and DW Verzi. A Simple 1-D Physical Model for the Crawling Nematode Sperm Cell. Journal of Statistical Physics, 110(3-6), 2003.

[11] T.M. Roberts and K.L. King. Centripetal flow and directed reassembly of the major sperm protein(MSP) cytoskeleton in the amoeboid sperm of the nematode, Ascaris suum. Cell Motility and the Cytoskeleton, 20(3):228-241, 1991.

[12] T.M. Roberts and M. Stewart. Nematode sperm locomotion. Current Opinion in Cell Biology, 7(1):13-17, 1995.

[13] T.M. Roberts and M. Stewart. Acting Like Actin The Dynamics of the Nematode Major Sperm Protein (MSP) Cytoskeleton Indicate a Push-Pull Mechanism for Amoeboid Cell Motility. The Journal of Cell Biology, 149(1):7-12, 2000.

[14] S. Sepsenwol and S.J. Taft. In vitro induction of crawling in the amoeboid sperm of the nematode parasite, Ascaris suum. Cell Motility and the Cytoskeleton, 15(2):99-110, 1990.

[15] C.W. Wolgemuth, L. Miao, O. Vanderlinde, T. Roberts, and G. Oster. MSP Dynamics Drives Nematode Sperm Locomotion. Biophysical Journal, 88(4):2462-2471, 2005.

[16] K. Yi, S.M. Buttery, M. Stewart, and T.M. Roberts. A Ser/Thr Kinase Required for Membrane-associated Assembly of the Major Sperm Protein Motility Apparatus in the Amoeboid Sperm of Ascaris. Molecular Biology of the Cell, 18(5):1816, 2007.

[17] M. Zajac, B. Dacanay, W.A. Mohler, and C.W. Wolgemuth. Depolymerization-driven flow in nematode spermatozoa relates crawling speed to size and shape. Biophysical Journal, 2008. 


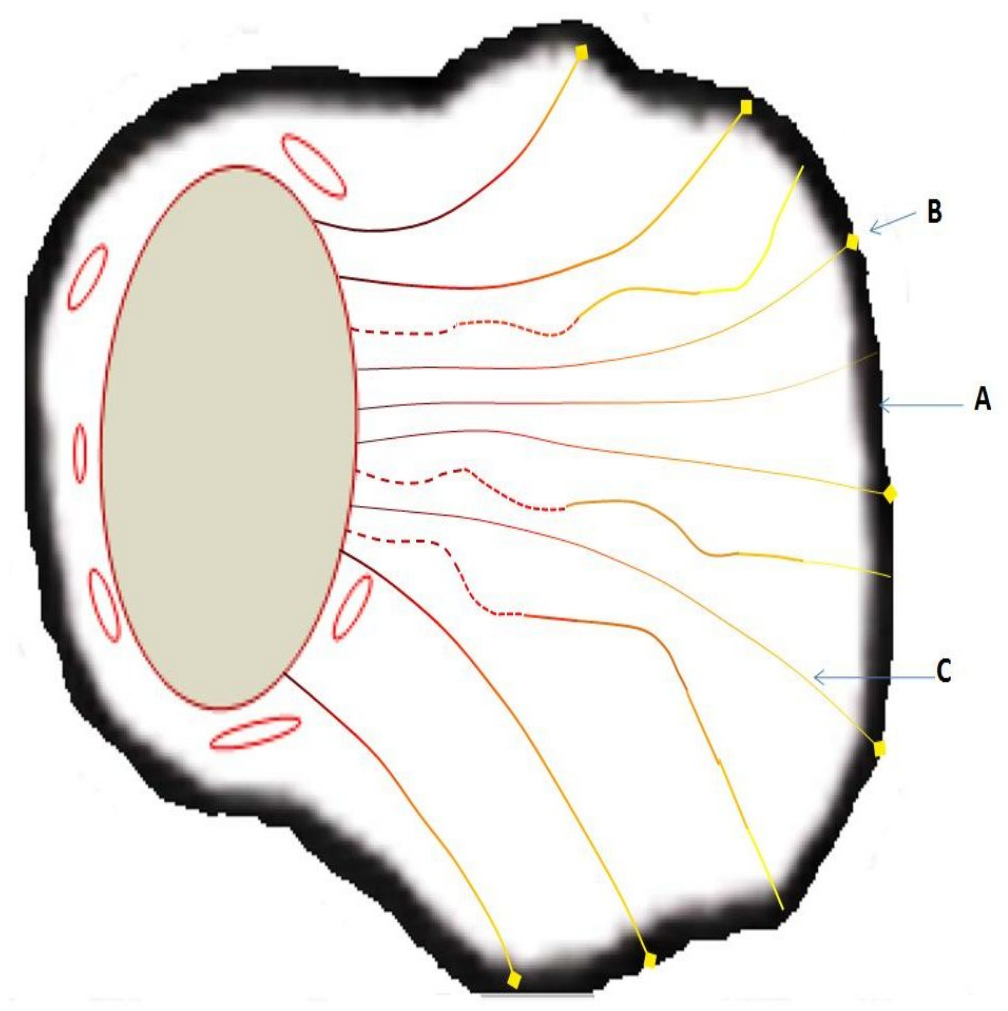

Figure 1:

Top View of Nematode Sperm Cell

A schematic showing some critical structures of the Ascaris suum cytoskeleton. (A) points to the leading edge of the lamellipod. (B) points to a membrane bound vesicle where the polymerization reactions take place. (C) points to a cytoskeletal bundle which are assembled at the leading edge and disassembled at the rear of the cell. 


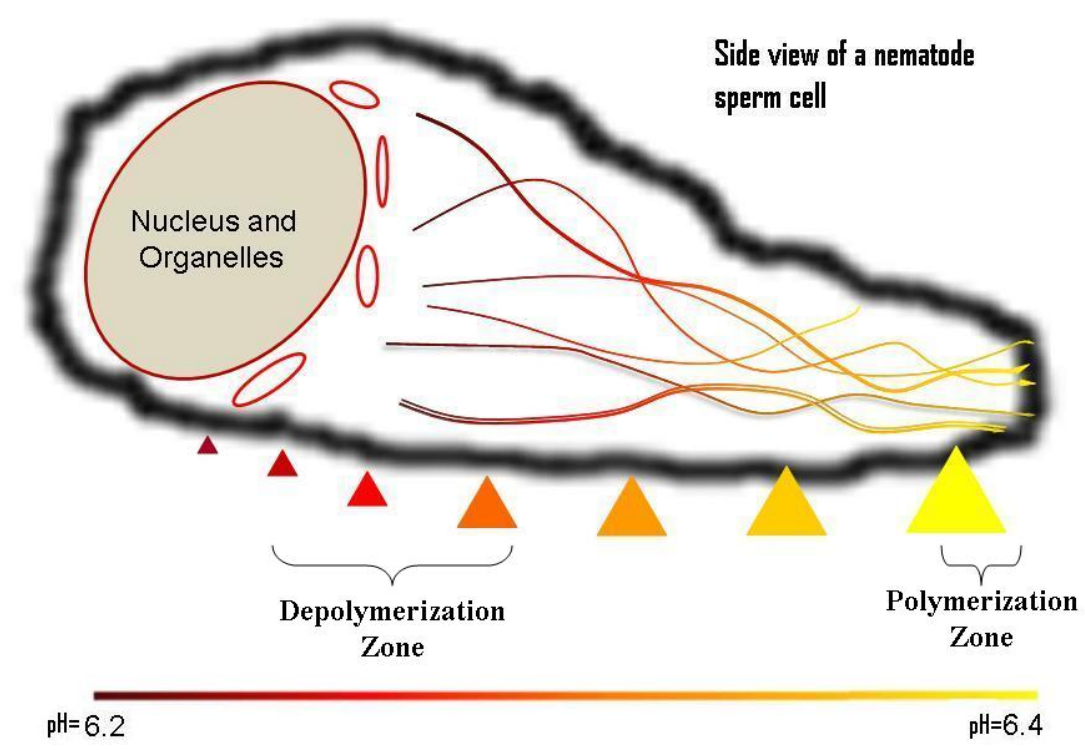

Figure 2: A side view of a nematode sperm cell showing the spatial separation of the processes of protrusion/polymerization and contraction/depolymerization. The triangles along the bottom of the figure illustrate the decreasing strength of adhesion as the $\mathrm{pH}$ decreases. 


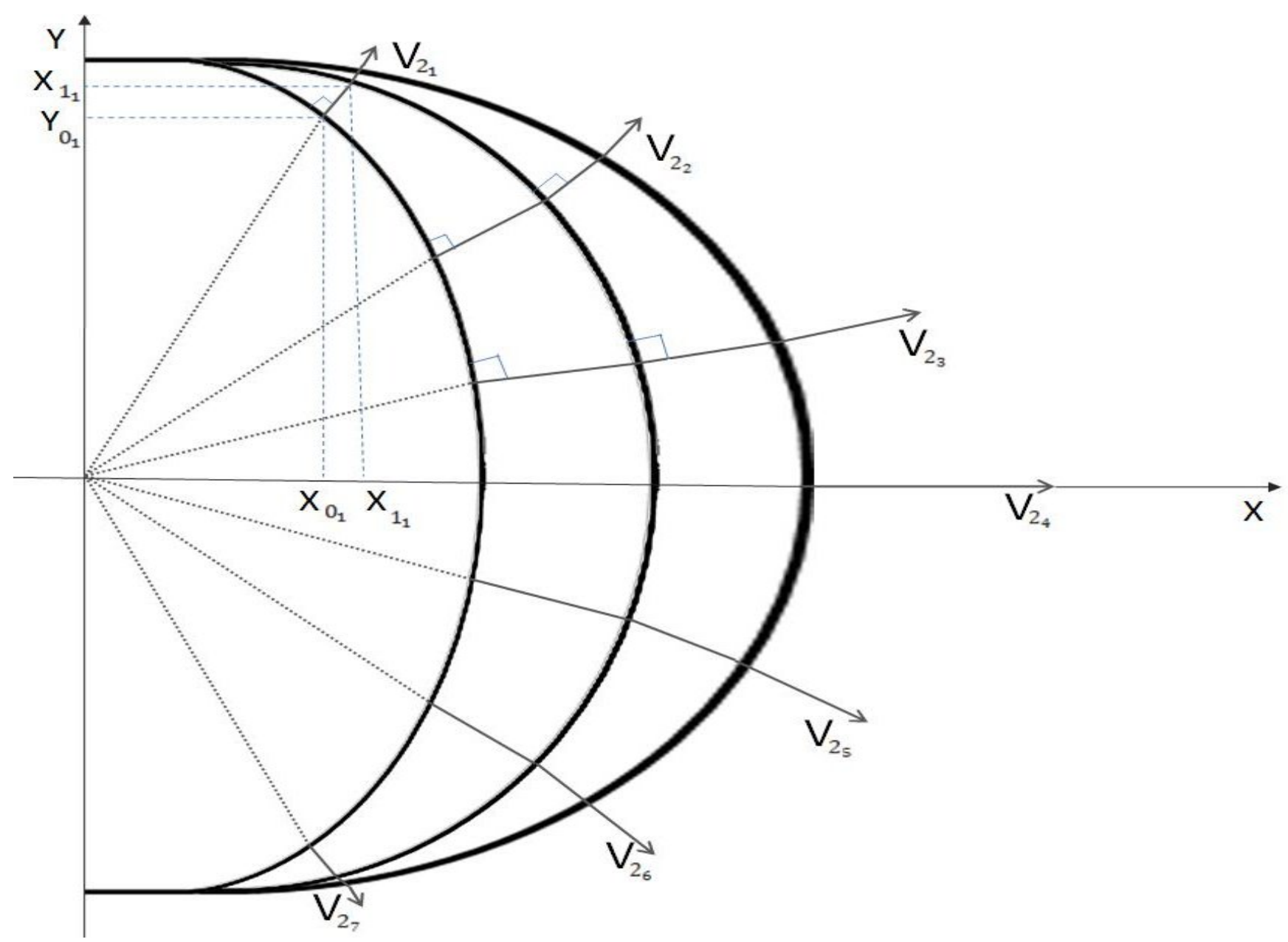

Figure 3:

\section{Geometry of Protrusion}

Our model uses difference equation (4) to model the position of bundles through time. This image shows initial rays used to calculate initial points on ellipse (our difference equations initial condition) where protrusion will begin. Protrusion begins from the set of points $\left(x_{0_{i}}, y_{0_{i}}\right)$. Above, points $\left(x_{0_{1}}, y_{0_{1}}\right)$ and $\left(x_{1_{1}}, y_{1_{1}}\right)$ show points of protrusion for bundle 1 for time $=0$ and 1 , respectively. Also, protrusion velocity vectors $v_{2_{i}}$ for bundles $i=1-7$ at time $=2$ are shown. Notice that bundles realize a curvature after the initial condition, as observed in laboratory videos. 


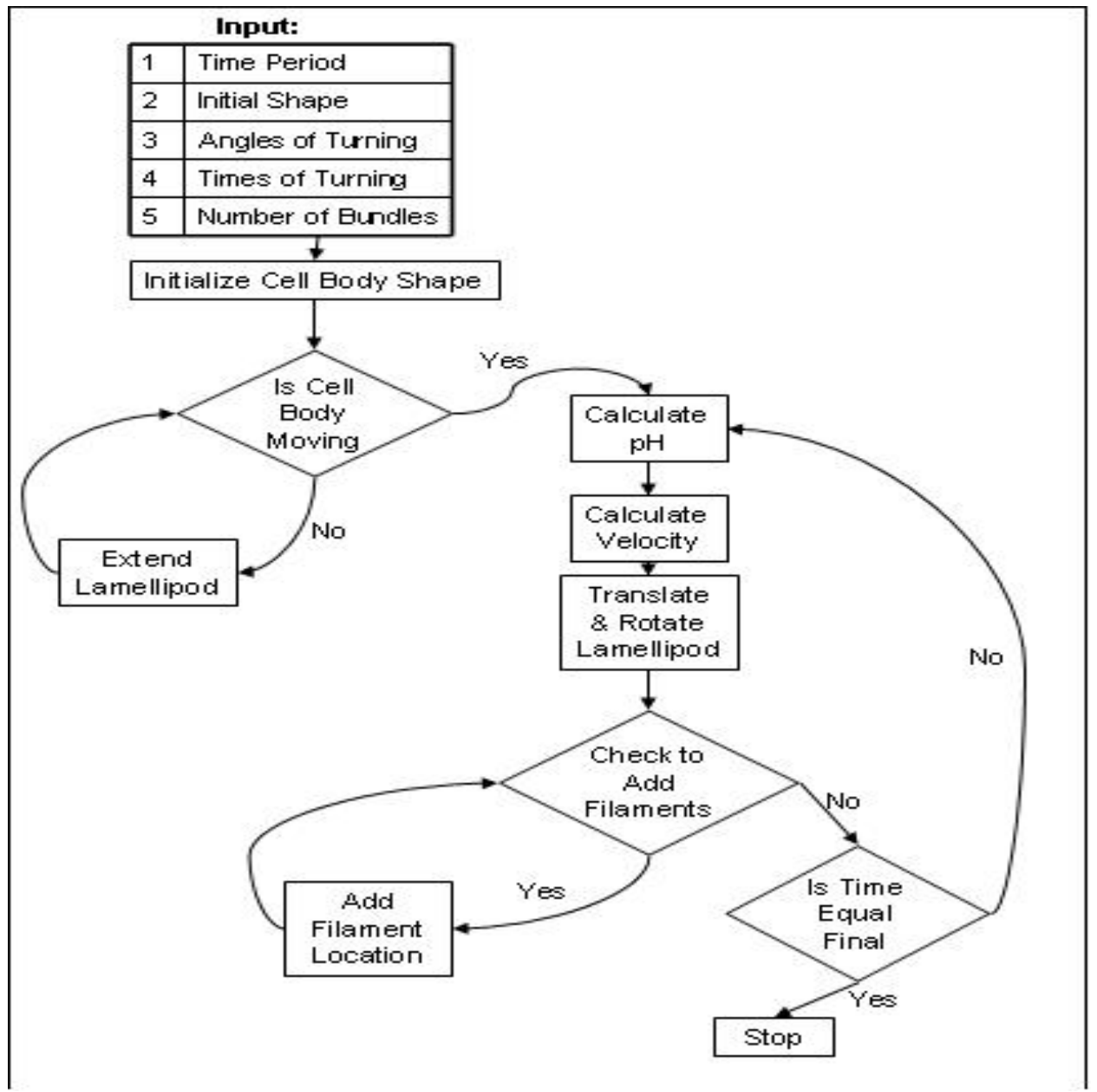

Figure 4:

\section{Computer Flow Chart for the Matlab Simulated Program}

This image shows the process of the Matlab simulated program, that begins with the user inputting variables, the program determining when the cell body begins movement, calculating $\mathrm{pH}$, translating the cell body in the direction of the $\mathrm{pH}$ gradient, adding filaments when necessary, and finally turning the cell body if indicated initially by the user. 

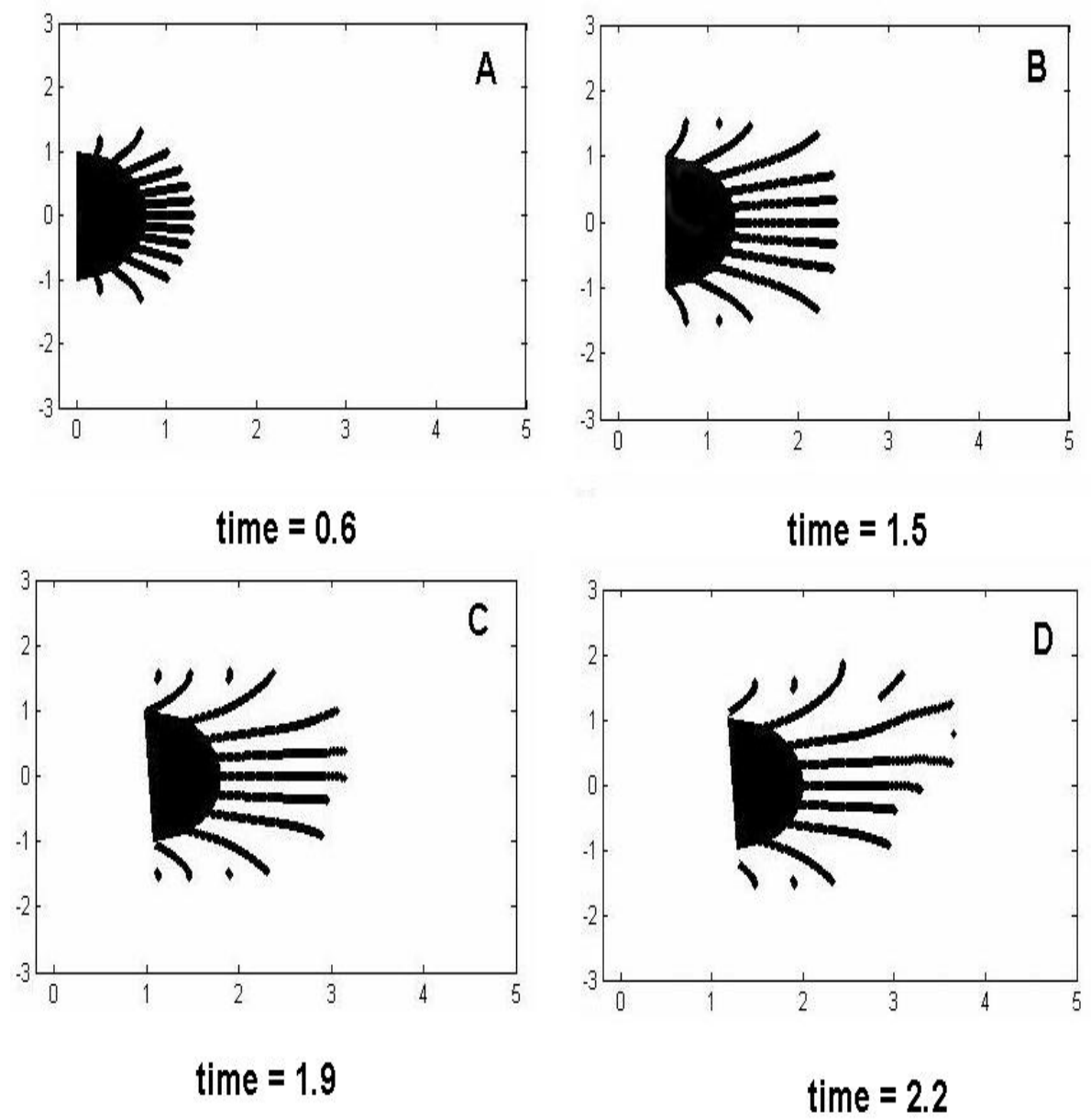

Figure 5:

\section{Turning of the Cell Body}

The image shows a computer simulation for the cell turning at time steps 0.6, 1.5, 1.9, 2.2. In (A), the lamellipod is protruding, but the cell body is still at the same position as in the beginning. In (B), the cell body movement has been triggered and both the front is protruding and the back is being moved due to the contraction. In $(\mathrm{C})$, the $\mathrm{pH}$ gradient has been changed and the cell starts turning. (D) completes the turn. 


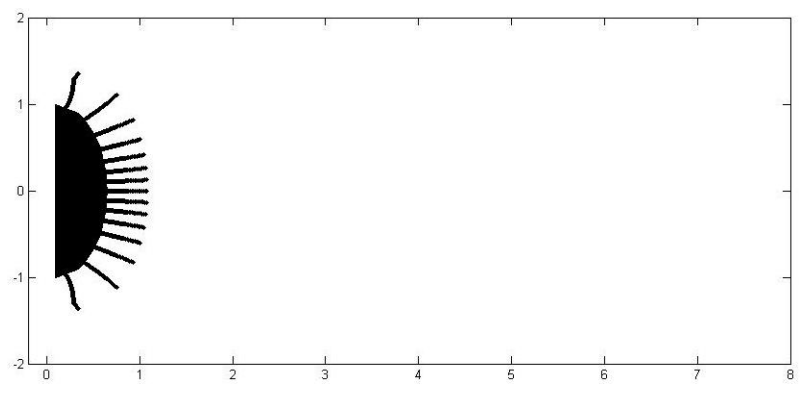

Figure 6: time $=0.5$

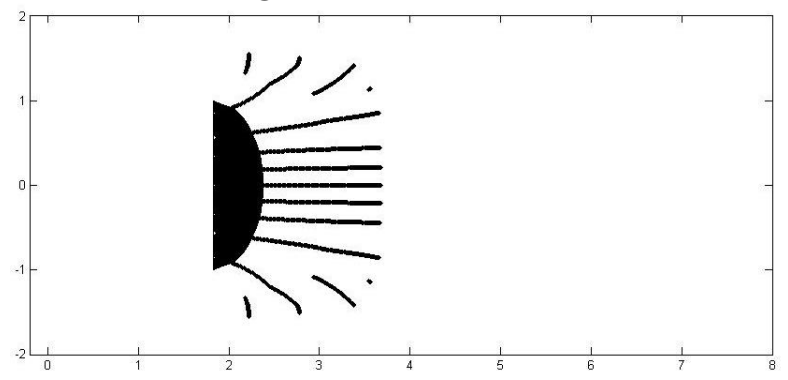

Figure $7:$ time $=2.5$

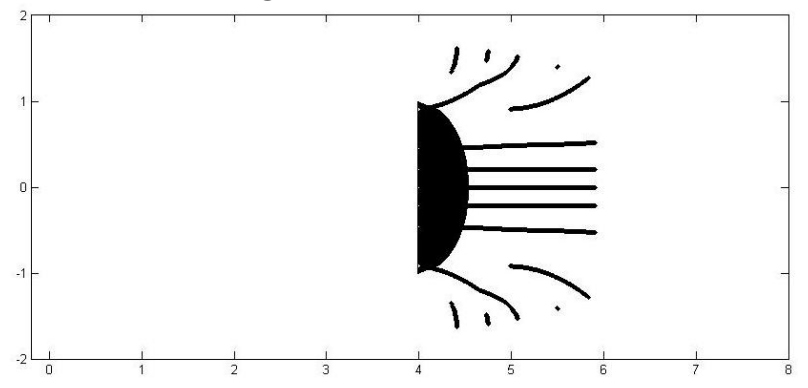

Figure 8: time $=5.0$

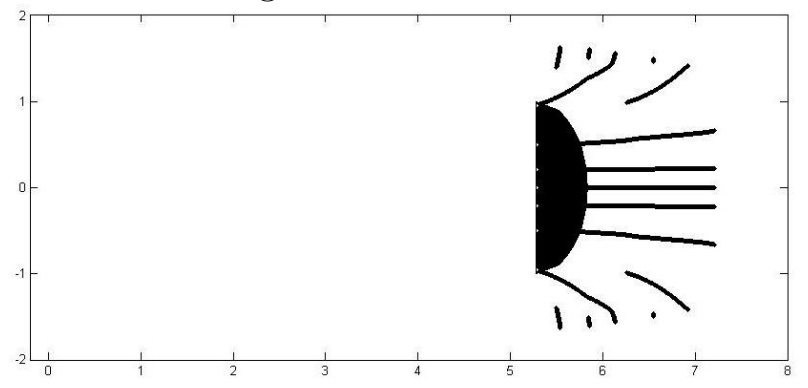

Figure 9: time $=6.5$

Figure 10:

\section{Steady Shape Cell Body Motion}

This image shows the steady shape cell body shape for a mid-ranged curved cell body $(\mathrm{k}=0.5)$ for time $=0.5$ to time $=6.5$, created using the Matlab simulated program. 

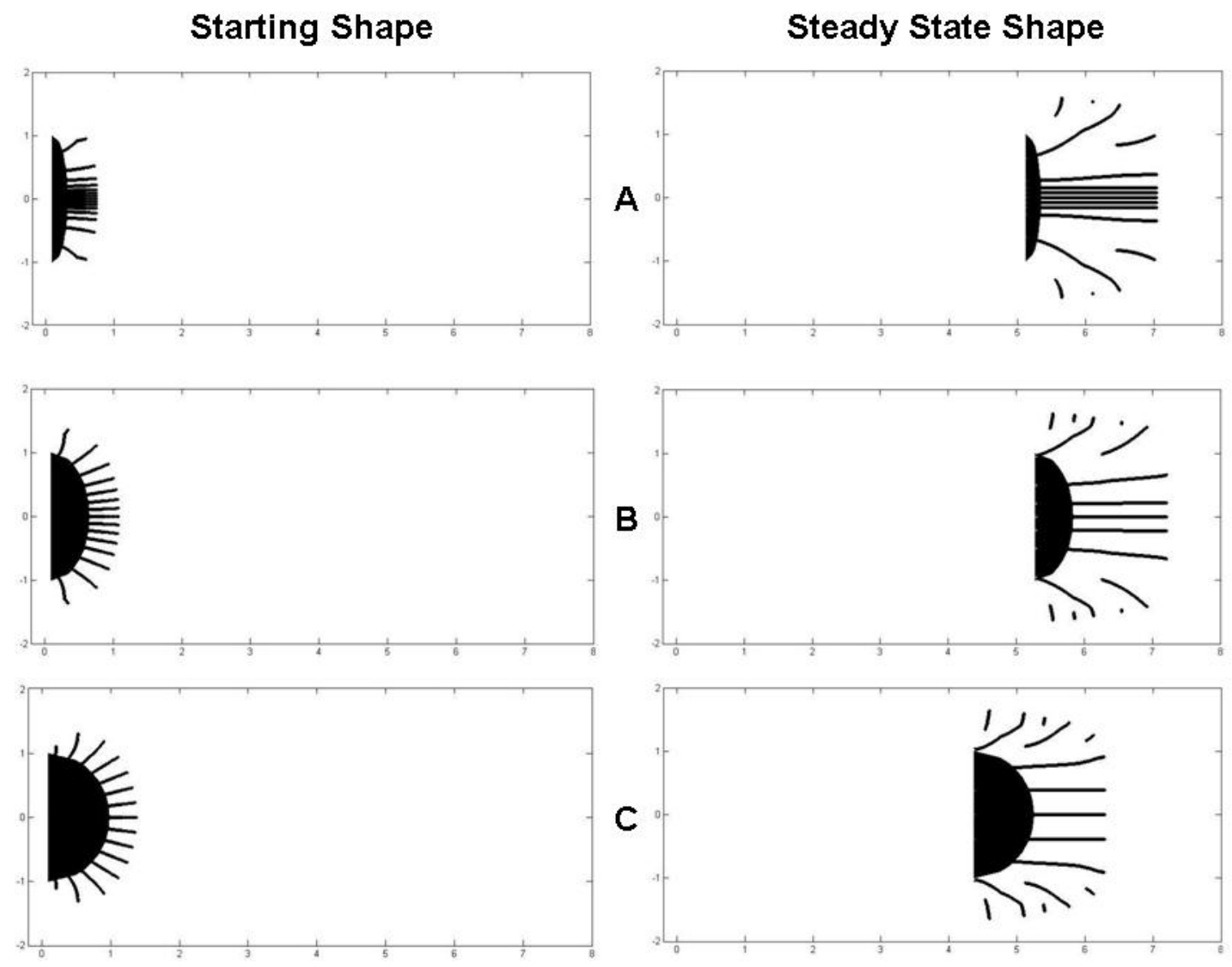

Figure 11: This image shows a consistant steady state shape reached for varying initial cell body shapes. (A) A very flat cell body shape, coefficient $=0.2$ (B) A midrange curved cell body, coefficient $=0.5(\mathrm{C})$ A very curved cell body shape, coefficient $=0.8$ 